\title{
Nutrition and physical activity recommendations for cancer survivors in Scotland: feasibility of a short course to promote behaviour change.
}

MASSON, L.F., DOUGLAS, F., MACLURE, K.

MASSON, L.F., DOUGLAS, F. and MACLURE, K. 2020. Nutrition and physical activity recommendations for cancer survivors in Scotland: feasibility of a short course to promote behaviour change. Nutrition Bulletin, 45(1), pages 66-73, which has been published in final form at https://doi.org/10.1111/nbu.12419. This article may be used for non-commercial purposes in accordance with Wiley Terms and Conditions for Use of SelfArchived Versions. 


\section{Title}

Nutrition and physical activity recommendations for cancer survivors in Scotland: feasibility of a short course to promote behaviour change

\section{Running head}

Nutrition for cancer survivors in Scotland

\section{Authors}

L.F. Masson, F. Douglas and K. MacLure

\section{Authors' affiliations}

Robert Gordon University, Aberdeen, Scotland.

\section{Corresponding author}

Dr Lindsey F Masson

Lecturer in Nutrition

School of Pharmacy and Life Sciences

Robert Gordon University

Garthdee Road

Aberdeen

AB10 7GJ

Scotland

Email: I.f.masson@rgu.ac.uk 


\section{Abstract}

More people are living for longer following a cancer diagnosis, however long-term survivors are more likely to experience chronic illnesses. Improving their diet and physical activity behaviours may increase survival and reduce the risk of cancer recurrence and other non-communicable diseases. The World Cancer Research Fund and American Institute for Cancer Research recommend that cancer survivors aim to be a healthy weight and physically active; eat a diet rich in wholegrains, vegetables, fruits and beans; limit consumption of 'fast foods', red and processed meat, sugar sweetened drinks and alcohol; and meet nutritional needs through diet alone rather than relying on supplements. Evidence suggests that cancer survivors are receptive to receiving advice and making dietary and physical activity changes, but barriers to improving the diet and being physically active need to be explored and addressed. We collaborated with CLAN Cancer Support (an independent charity) to assess the feasibility of a two-day course designed to improve diet and physical activity in cancer survivors in Scotland. Further, it explored the barriers and facilitators that cancer survivors identify in relation to eating a healthy diet and being physically active. The course included presentations, practical activities and group discussions. Initial analysis indicates that factors specific to this population need to be designed into the delivery of the course to enhance recruitment and promote behaviour change. Research then needs to be translated into sustainable support programmes accessible by all cancer survivors. This article describes the rationale behind the study, its design and expected outcomes.

\section{Keywords}

Nutrition, diet, physical activity, cancer, healthy eating 


\section{Cancer survivorship and the role of nutrition and physical activity}

Since the early 1990s, cancer incidence rates have increased by $12 \%$ in the UK (Cancer Research UK 2019). The UK incidence rate exceeds that of two-thirds of Europe (rank 11 of 40), and within the UK, Scotland has a higher incidence rate of all cancers, excluding non-melanoma skin cancer, than England or Northern Ireland: 606.8 versus 593.2 or 590.7 per 100,000 respectively in 2016 (Cancer Research UK 2019). Nevertheless, improvements in cancer detection and treatment have increased cancer survival significantly in Scotland in recent years. The five-year relative survival for individuals aged $15-99$ years increased from $36.6 \%$ to $53.6 \%$ for those diagnosed between 1987-1991 and 2007-2011 respectively, although these figures vary considerably by cancer type: $3.8 \%$ for pancreatic cancer to $98.7 \%$ for testicular cancer (ISD Scotland 2019).

Whilst more people are living for longer following a cancer diagnosis, long-term survivors are more likely to experience chronic illnesses. A British cohort of 26,213 individuals who had survived at least five years following a cancer diagnosis were more likely to develop osteoporosis compared with controls without cancer; adjusted hazard ratios $(\mathrm{HR})$ were $2.49(95 \%$ confidence interval $(\mathrm{Cl}): 1.93,3.22), 1.41(95 \% \mathrm{Cl}: 1.15,1.73)$ and 1.26 $(95 \% \mathrm{Cl}: 1.13,1.40)$ in prostate, colorectal and breast cancer survivors respectively (Khan et al. 2011). In addition, breast cancer survivors had a higher incidence of heart failure (adjusted $\mathrm{HR}=1.95 ; 95 \% \mathrm{Cl}: 1.27,3.01$ ) and coronary artery disease (adjusted $\mathrm{HR}=1.27 ; 95 \% \mathrm{Cl}: 1.11,1.44$ ), and colorectal cancer survivors had a higher incidence of dementia (adjusted $\mathrm{HR}=1.68 ; 95 \% \mathrm{Cl}: 1.20,2.35$ ) and diabetes (adjusted $\mathrm{HR}=1.39 ; 95 \%$ Cl: 1.12, 1.72). The higher risk of chronic illnesses was attributed to cancer treatments (e.g. radiotherapy, chemotherapy and hormone treatment) and/or underlying lifestyle factors such as diet and physical activity (Khan et al. 2011).

The World Cancer Research Fund (WCRF) and American Institute for Cancer Research (AICR) suggest that improving diet and physical activity behaviours may increase cancer survival and reduce the risk of cancer and other non-communicable diseases, including obesity, type 2 diabetes mellitus, coronary heart disease and stroke (WCRF/AICR 2018a). They advise cancer survivors to follow their recommendations for cancer prevention (Table 1). This includes being a healthy weight and physically active; eating a diet rich in wholegrains, vegetables, fruits and beans; limiting consumption of 'fast foods', red and processed meat, sugar sweetened drinks and alcohol; and aiming to meet nutritional needs through diet alone rather than relying on supplements (WCRF/AICR 2018a). These recommendations are similar to UK and Scottish dietary guidelines, except for 
processed meat. For cancer prevention and survival, it is recommended to "consume very little, if any, processed meat" and to limit consumption of red meat to about 350-500 g/week (WCRF/AICR 2018a). However, the UK Eatwell Guide and Scottish Dietary Goals combine red and processed meat, with a recommended intake towards the higher end of the range given by WCRF/AICR: no more than $70 \mathrm{~g} / \mathrm{day}$ ( $490 \mathrm{~g} / \mathrm{week}$ ) of red or processed meat (Public Health England 2016; Scottish Government 2016).

The benefit of the WCRF/AICR (2018a) recommendations for cancer survivors is supported by systematic reviews and meta-analyses which have explored the association between diet, physical activity and risk of mortality and cancer recurrence. Firstly, a systematic review and meta-analysis of 117 cohort studies enrolling 209,597 cancer survivors found significant associations between diet postdiagnosis and overall mortality. There was an increased risk ratio (RR) with the highest category of a Western dietary pattern (RR=1.51;95\% $\mathrm{Cl}$ : 1.24 , $1.85)$ and alcohol intake $(R R=1.31 ; 95 \% \mathrm{Cl}: 1.04,1.66)$, and a reduced risk with the highest category of a "prudent/healthy dietary pattern" $(\mathrm{RR}=0.77 ; 95 \% \mathrm{Cl}: 0.60,0.99)$, compared to the lowest category of consumption (Schwedhelm at al. 2016). Similarly, a systematic review of 38 studies found that risk of overall mortality and death from other causes in breast cancer survivors was reduced by adhering to "a high-quality diet" and "prudent diet" after diagnosis, and increased by a Western diet before and after diagnosis (Jochems et al. 2018). Postdiagnosis alcohol intake has also been associated with cancer recurrence $(\mathrm{RR}=1.31 ; 95 \% \mathrm{Cl}$ : 1.04, 1.66 for the highest versus lowest intake category) (Schwedhelm et al. 2016), and reducing the amount of fat postdiagnosis appears to reduce the risk of breast cancer recurrence (Jochems et al. 2018). Another review of 43 observational studies found that a low-fat, high-fibre diet might protect against cancer recurrence and progression, and there was a dose-response relationship between physical activity and better outcomes (Davies et al. 2011).

It has been suggested that the mechanism behind the benefit of diet and physical activity on improved survival relates to body weight. However, two recent systematic reviews state that there is currently a lack of both observational and experimental evidence to support the suggestion that weight loss improves clinical outcomes for overweight cancer survivors, and findings from well-designed randomised controlled trials that are now underway are not yet available (Jackson et al. 2017; Chlebowski and Reeves 2018). Such interventions need to be long-term (i.e. at least 1-2 years), and be able to sustain participant motivation and engagement throughout the intervention and after it has ended in order to optimise weight loss and weight maintenance. This lack of 
strong evidence is consistent with findings from the only WCRF/AICR report on cancer survivors (2018b) which found limited, but suggestive, evidence for better survival after breast cancer with having a healthy body mass index $\left(18.5-24.9 \mathrm{~kg} / \mathrm{m}^{2}\right)$, in addition to being physically active, eating foods containing fibre and soy, and having a lower intake of total fat and saturated fat. There was insufficient evidence to make specific recommendations for breast cancer survivors due to limitations in the design or execution of the available evidence. Therefore, current recommendations for cancer survivors are the same as those for cancer prevention.

Although the recommendations for cancer prevention and survival are broadly consistent with general healthy eating advice, a recent review of 51 studies including 2,620,586 adult cancer survivors indicated that adherence to the WCRF/AICR recommendations is low (Tollosa et al. 2019). Whilst a pooled estimate of $83 \%$ had low or no alcohol intake, only $31 \%$ achieved $\geq 21 \mathrm{~g} /$ day of fibre or wholegrain. Adherence to multiple dietary behaviours ranged from $7 \%$ to $40 \%$ in 13 studies, and was better, but still low, in recent ( $\leq 5$ years) versus long-term ( $>5$ years) survivors (pooled estimate of $31 \%$ versus $25 \%$ ).

\section{Nutrition and physical activity in cancer survivors in Scotland}

The Scottish population as a whole is failing to meet goals for consumption of fruit and vegetables and oily fish, and for intakes of total fat, saturated fat, free sugars and fibre, and there has been little change in these dietary behaviours between 2001 and 2015 (Barton et al. 2018). Similarly, the 2018 Scottish Health Survey found no change in the mean number of portions of fruit and vegetables consumed per day between 2003 (3.1) and 2018 (3.2), or in the proportion of adults meeting the recommended 5-a-day intake between 2003 (21\%) and 2018 (22\%) (Hinchliffe 2019). In addition, two thirds (65\%) of adults in Scotland are overweight (including obese) and $28 \%$ are obese, and there has been no change in these figures since 2008 (Dougall 2019). Whilst two thirds $(66 \%)$ of adults are meeting guidelines for moderate or vigorous physical activity, these figures decline with age to $53 \%$ in those aged $65-74$ years, and $31 \%$ in those aged 75 years and above (Mirani 2019). Finally, $24 \%$ of adults in Scotland are drinking alcohol at hazardous or harmful levels, i.e. over 14 units/week (Vosnaki 2019).

Little is known about the nutritional status of cancer survivors post-treatment in Scotland, although analysis of 16,282 Scottish Health Survey respondents from 1995, 1998, 2003 and 2008 revealed that cancer survivors were significantly more likely to eat at least five portions/day of fruit and vegetables $(21 \%)$ versus those with no cancer history (15\%), but less likely to engage in at least two hours/week of physical activity (35\% versus $45 \%)$. 
However, the prevalence of obesity was similar ( $27 \%$ versus $29 \%$ ), as was the proportion drinking more than 21 (men) or 14 (women) units per week of alcohol (16\% versus 18\%) (Wang et al. 2015).

A small study of 74 cancer survivors in Scotland has suggested that individuals may make positive changes to their diet following a cancer diagnosis (Masson et al. 2018). At least one quarter of participants reduced their frequency of consumption of red meat $(27 \%)$; processed meat $(37 \%)$; sweet biscuits and chocolate biscuits (30\%); buns, sweet pastries, scones, flapjacks, croissants and doughnuts $(26 \%)$; chocolates and chocolate bars (30\%); and crisps and other packet snacks $(28 \%)$. In addition, $89 \%$ agreed that it was important for them to follow a healthy diet, and $76 \%$ agreed that they would consider improving their diet. Similarly, focus groups carried out in Scotland and England revealed that colorectal cancer survivors would welcome dietary advice following treatment, with many participants actively seeking lifestyle guidance (Anderson et al. 2013). These results are consistent with the conclusion of a systematic review of social cognitive theory-based physical activity and/or nutrition behaviour change interventions that "cancer survivors are willing and able to make improvements to diet" (Stacey et al. 2015).

These findings support the hypothesis that a cancer diagnosis may be a "teachable moment", representing an opportunity to introduce interventions that result in higher than expected positive health-related behaviour changes (Lawson and Flocke 2009), i.e. at a time when individuals may be particularly receptive to advice and motivated to make changes. However, an English study has highlighted that a cancer diagnosis may not necessarily represent a "teachable moment" for everyone (Corbett et al. 2018). Although some individuals make positive lifestyle changes following treatment, others may not engage with diet and physical activity recommendations. Reasons for a lack of engagement vary, including a belief that behaviour change is unnecessary or undesirable, uncertainty about how to implement recommendations for improvement, or a perceived lack of support from health care providers (Corbett et al. 2018). Therefore, in addition to providing information on recommendations, interventions to improve diet and physical activity in cancer survivors must address barriers to implementing recommendations.

\section{Interventions to improve nutrition and physical activity in cancer survivors}

Most interventions to improve diet and/or physical activity in cancer survivors have been carried out in breast cancer survivors, with the mode of delivery including face-to-face counselling (individual/single or group 
sessions) and broad-reach interventions delivered via telephone, text messages, social media (e.g. Facebook), online modules or mailed print materials. Interventions have focussed on physical activity, specific or multiple dietary components, or a combination of diet and physical activity. Dietary interventions most commonly focus on fruit and/or vegetable intake, fat, fibre, diet quality or weight control. Intervention length varied from 5 weeks to 4 years, and time since diagnosis also varied or was not reported (Goode et al. 2015; Stacey et al. 2015; Roberts et al. 2017).

Recruitment, adherence and engagement/follow-up have been identified as significant problems with such trials (Koutoukidis et al. 2017; Roberts et al. 2017), and barriers to participation need to be minimised. For example, in a 24-week behavioural lifestyle programme for endometrial cancer survivors in the UK, inconvenience and transport or distance to the trial site were cited as the most common barriers to participation among eligible survivors who declined to participate (Koutoukidis et al. 2017). Barriers to adherence to healthy behaviours can exist at an individual (e.g. lack of time), social (e.g. family obligations) or environmental (e.g. perceived cost of fresh fruit and vegetables) level. Such barriers may apply to the general population or be specific to cancer survivors, such as taste changes related to treatment. For example, amongst 97 breast cancer survivors, individual level barriers to physical activity and a healthy diet included lack of time and lack of motivation, but also physical symptoms such as pain and fatigue (Cho and Park 2018). Therefore, barriers at multiple levels, and barriers specific to cancer survivors (and potential solutions), need to be further explored and addressed to improve dietary and physical activity behaviours.

Interventions in cancer survivors are more complex to implement, analyse and compare versus interventions for cancer prevention due to the variability between participants (in cancer site, treatment type, time since diagnosis etc.), and heterogeneity in study design (described above), sample size and outcomes measured. Overall, broad-reach lifestyle interventions have been found to be effective (Goode et al. 2015), and interventions based on social cognitive theory found to "demonstrate promise" in improving dietary and physical activity behaviours (Stacey et al. 2015). A more recent systematic review of interventions using digital technologies (e.g. text messaging, email, mobile applications, video-conferencing, social media and/or websites) in cancer survivors concluded that they may improve physical activity and reduce body mass index, although there was mixed evidence for diet (Roberts et al. 2017). Future studies need to consider the translation 
of all this research into effective and sustainable support programmes that can be delivered and maintained outwith research interventions.

\section{Research aims and study design}

Evidence indicates that (i) the Scottish diet needs to improve and such improvements would be beneficial for cancer survivors, (ii) cancer survivors will be receptive to receiving advice and making dietary and physical activity changes, and (iii) barriers to improving the diet and being physically active need to be further explored in this group so that strategies for overcoming them can be developed. However, the nature of such an intervention to improve diet and physical activity needs to be convenient and attractive to potential participants and relevant to their needs in order to promote successful recruitment and follow-up and an effective intervention. Therefore, we aimed to test the feasibility of a two-day course to improve diet and physical activity behaviours in cancer survivors in Scotland, and to explore the barriers and facilitators that cancer survivors identify in relation to eating a healthy diet and being physically active.

A 'feasibility study' is conducted to find out "can this study be done?" (WCRF International 2018), and as such our expected outcomes included (i) factors that will affect recruitment and follow-up in the main study, i.e. recruitment (level of interest), most effective method of advertisement, preferred days for attending, feedback on course acceptability and satisfaction, and follow-up response rates, and (ii) knowledge of barriers and potential solutions to eating healthily and being physically active in cancer survivors to be addressed during the main follow-on study. This study was approved by Robert Gordon University's School of Pharmacy and Life Sciences Ethics Review Panel (S176, 29th January 2019).

The study employed a mixed-methods theory-based participatory approach and was carried out in collaboration with CLAN (originally Cancer Link Aberdeen and North) Cancer Support - an independent charity that offers services free-of-charge to anyone affected by any type of cancer, whether affected personally, as a carer, family member or close friend. CLAN works across the North-East of Scotland, Moray, Orkney and Shetland, and offers information and support, counselling, complementary therapies, social and wellbeing activities, support for children and families, and affordable accommodation at CLAN Haven in Aberdeen alongside assistance with transport to and from hospital (CLAN Cancer Support 2019). Our longer-term aim is to develop course materials on diet and physical activity that can be delivered by CLAN staff/volunteers as part of their regular services, 
thus contributing to the sustainability of the provision of diet and lifestyle advice to their users. As such, our study helps to address recommendations for research to inform integration within clinical care, centralised survivorship clinics, and community-based health care organisations, e.g. local charities (Goode et al. 2015).

We recruited men and women aged $\geq 18$ years who had received a diagnosis of cancer and completed treatment via CLAN Cancer Support (including leaflets at CLAN centres and details on their Facebook page and website) and local media (newspapers and radio). Participation was not restricted to individuals with a specific cancer (e.g. all breast cancer) because the WCRF/AICR nutrition and physical activity recommendations apply to all cancer survivors, and we wanted the course materials to be available for use by CLAN in the future with no restrictions based on type of cancer. Those who were underweight or following specific dietary advice from their doctor or dietitian were not eligible to participate. Eligible individuals were invited to attend the two-day course 'EatWell @CLAN' in Aberdeen, Elgin or Kirkwall. We aimed to recruit 10-14 participants per course which would run twice at each centre (maximum 84 participants).

The course was based on WCRF/AICR's diet and physical activity recommendations (2018a) and the COM-B (Capability, Opportunity and Motivation for Behaviour) model which links to the Behaviour Change Wheel (BCW) for targeting behaviour change interventions (Michie et al. 2011). The BCW considers Intervention Functions (education, persuasion, incentivisation, coercion, training, restriction, environmental restructuring, modelling, and enablement) and Policy Categories (communication/marketing, guidelines, fiscal, regulation, legislation, environmental/social planning, and service provision). The course was created in discussion with CLAN staff following a review of written and verbal feedback from participants of a weight management programme (Counterweight Project Team 2008) which was delivered at CLAN House in Aberdeen in 2017 and 2018. The course was also conceptualised to be as participative and facilitative as possible for two reasons: (i) to encourage participants to reflect on their lived experiences and knowledge and share that with their respective groups and course leaders, and (ii) to support active as opposed to passive learning. CLAN advised on the length and timing of the course that they felt would be most convenient for their clients. The two days were designed to take place one week apart, from 10am to $3.30 \mathrm{pm}$.

The course included a mix of PowerPoint presentations, practical activities and group discussions (Table 2). Practical activities included recalling various colours of vegetables and fruit consumed in the past week; 
examining, interpreting and comparing food labels on similar products; and classifying examples of meat and fish as red or processed meat, and oily or white fish. The first practical activity involved using craft materials to create a "Rainbow warrior" - an object that participants could take home to act as a reminder of the course and advice provided. The purpose of this activity was to encourage the participants to chat informally and relax. Qualitative data was collected as part of discussions on barriers and solutions to making healthier choices, and changes in dietary preferences post-treatment. For example, use of a 'talking wall' involved asking participants to individually write down and share ideas, then as a group to classify them into themes. A rainbow theme linked both days of the course to highlight the importance of variety in the diet (e.g. different colours of vegetables and fruit) and variety in physical activities (i.e. aerobic, resistance/strength, flexibility and balance exercises).

The course was delivered in March and April 2019 by a Registered Nutritionist listed on the UK Voluntary Register of Nutritionists (Association for Nutrition 2019) and a qualitative health services researcher in collaboration with CLAN. The presentations and practical activities were delivered by the Registered Nutritionist whilst group discussions were facilitated by the qualitative researcher.

When participants arrived for the course, they were asked to sign a consent form and complete a baseline questionnaire about their diet and physical activity habits. Each participant was provided with a course booklet which included a copy of the PowerPoint slides, plus copies of the UK Eatwell guide and factsheets available from websites for The Association of UK Dietitians, British Nutrition Foundation, World Cancer Research Fund, Macmillan Cancer Support, and Department of Health and Social Care. At the end of each day of the course, participants were asked to complete an evaluation form to provide feedback on course acceptability and satisfaction using Likert scale attitudinal items and open-ended questions. Three months later (in June and July 2019), participants were mailed a follow-up questionnaire regarding their diet and physical activity habits, to be returned by post.

\section{Eligibility and participation}

Of 27 people (4 men and 23 women) who expressed an interest in the study, two men and nine women met the eligibility criteria and were able to attend the course (Table 3). Reasons for the low participation rate included: (i) ineligibility (some interested individuals were still receiving chemotherapy or had not received a cancer diagnosis), (ii) dates of the course did not suit (reasons given included 'on holiday', 'not enough notice' or 'can't 
get time off work'), (iii) did not want to attend alone and wanted to attend only if they could bring their spouse, and (iv) concern about fitting in with the group - one young female was concerned she would feel 'out of place' at the course, as CLAN events tend to include older individuals (figure 1). We are currently analysing the data collected from questionnaires, evaluation forms, group discussions and feedback from CLAN staff and volunteers.

\section{Conclusion}

'EatWell@CLAN' is a two-day course designed to improve diet and physical activity in cancer survivors in Scotland. Initial analysis of this feasibility study indicates that factors specific to this population group need to be designed into the delivery of the course to enhance recruitment and promote behaviour change. For example, in relation to the COM-B model, fatigue affects Capability to attend, and timings and work commitments affect the Opportunity to attend the course. Whilst participants were Motivated to make positive lifestyle changes, more must be done to explore barriers and facilitators to participation and improving diet and physical activity Behaviours in the wide spectrum of cancer survivors, and to assist targeted behaviour interventions in this group. Research then needs to be translated into sustainable support programmes which can be accessed by all cancer survivors.

\section{Acknowledgements}

Lindsey Masson drafted the manuscript, and Katie MacLure and Flora Douglas reviewed the manuscript and contributed to revisions prior to submission. This work received funding from Robert Gordon University. The authors acknowledge and thank the staff and volunteers of CLAN Cancer Support for their support, in particular Colette Backwell, Pamela Jack, lona Mitchell, Elspeth Linklater, Karen Scott and Tracy Sellar. The authors also thank Susan Duthie and Marie Goua for critically reviewing the manuscript.

\section{Conflict of interest}

The authors have no conflict of interest to declare. 


\section{References}

Anderson AS, Steele R \& Coyle J (2013) Lifestyle issues for colorectal cancer survivors - perceived needs, beliefs and opportunities. Supportive Care in Cancer 21 (1): 35-42.

Association for Nutrition (2019) The UK Voluntary Register of Nutritionists (UKVRN). Available at http://www.associationfornutrition.org/default.aspx?tabid=76 (accessed 30 October 2019).

Barton KL, Masson LF \& Wrieden WL (2018) Estimation of food and nutrient intakes from food purchase data in Scotland: 2001-2015. Aberdeen: Food Standards Scotland. Available at http://www.foodstandards.gov.scot/downloads/D19-01_Final_Report_2001-2015_-_130618.pdf (accessed 5 October 2019).

Cancer Research UK (2019) Cancer statistics for the UK. Available at https://www.cancerresearchuk.org/health-professional/cancer-statistics-for-the-uk (accessed 5 October 2019).

Chlebowski RT \& Reeves MM (2016) Weight loss randomized intervention trials in female cancer survivors. Journal of Clinical Oncology 34 (35): 4238-4248.

Cho D \& Park CL (2018) Barriers to physical activity and healthy diet among breast cancer survivors: a multilevel perspective. European Journal of Cancer Care (Engl) 27 (1) doi: 10.1111/ecc.12772.

CLAN Cancer Support (2019) CLAN Cancer Support. Available at https://www.clanhouse.org/ (accessed 13 October 2019).

Corbett T, Cheetham T, Muller AM et al. (2018) Exploring cancer survivors' views of health behaviour change: "Where do you start, where do you stop with everything?" Psychooncology 27 (7): 1816-1824.

Counterweight Project Team (2008) Evaluation of the Counterweight Programme for obesity management in primary care: a starting point for continuous improvement. British Journal of General Practice 58: 548-554. 
Davies NJ, Batehup L \& Thomas R (2011) The role of diet and physical activity in breast, colorectal, and prostate cancer survivorship: a review of the literature. British Journal of Cancer 105 (Suppl 1): S52-73.

Dougall I (2019) Chapter 7: Obesity. In: McLean J \& Dean L (editors) The Scottish Health Survey 2018 edition. Volume 1. Main Report. The Scottish Government. Available at https://www.gov.scot/publications/scottish-health-survey-2018-volume-1-main-report/ (accessed 13 October 2019).

Goode AD, Lawler SP, Brakenridge CL et al. (2015) Telephone, print, and Web-based interventions for physical activity, diet, and weight control among cancer survivors: a systematic review. Journal of Cancer Survivorship 9 (4): 660-682.

Hinchliffe S (2019). Chapter 5: Diet. In: McLean J \& Dean L (editors) The Scottish Health Survey 2018 edition. Volume 1. Main Report. The Scottish Government. Available at https://www.gov.scot/publications/scottishhealth-survey-2018-volume-1-main-report/ (accessed 13 October 2019).

ISD Scotland (2019) Cancer Statistics. Available at https://www.isdscotland.org/HealthTopics/Cancer/Cancer-Statistics/All-Types-of-Cancer/ (accessed 6 October 2019).

Jackson SE, Heinrich M, Beeken RJ \& Wardle J (2017) Weight loss and mortality in overweight and obese cancer survivors: a systematic review. PLOS ONE 12 (1): e0169173.

Jochems SHJ, Van Osch FHM, Bryan RT et al. (2018) Impact of dietary patterns and the main food groups on mortality and recurrence in cancer survivors: a systematic review of current epidemiological literature. British Medical Journal Open 8 (2): e014530. doi:10.1136/ bmjopen-2016-014530.

Khan NF, Mant D, Carpenter L et al. (2011) Long-term health outcomes in a British cohort of breast, colorectal and prostate cancer survivors: a database study. British Journal of Cancer 105: S29-S37. 
Koutoukidis DA, Beeken RJ, Manchanda R et al. (2017) Recruitment, adherence, and retention of endometrial cancer survivors in a behavioural lifestyle programme: the Diet and Execise in Uterine Cancer Survivors (DEUS) parallel randomised pilot trial. British Medical Journal Open 7:e018015. doi:10.1136/bmjopen-2017018015.

Lawson PJ \& Flocke SA (2009) Teachable moments for health behaviour change: a concept analysis. Patient Education and Counselling 76 (1): 25-30.

Masson LF, Verra H, Cumming H et al. (2018) Changes in diet following a cancer diagnosis in Scotland. Proceedings of the Nutrition Society 77 (OCE1): E4.

Michie S, Van Stralen MM \& West R (2011) The behaviour change wheel: a new method for characterising and designing behaviour change interventions. Implementation Science 6: 42

Mirani K (2019). Chapter 6: Physical Activity. In: McLean J \& Dean L (editors) The Scottish Health Survey 2018 edition. Volume 1. Main Report. The Scottish Government. Available at https://www.gov.scot/publications/scottish-health-survey-2018-volume-1-main-report/ (accessed 13 October 2019).

Public Health England (2016) The Eatwell Guide. Public Health England. Available at http://www.gov.uk/government/publications/the-eatwell-guide (accessed 19 October 2019).

Roberts AL, Fisher A, Smith L et al. (2017) Digital heath behaviour change interventions targeting physical activity and diet in cancer survivors: a systematic review and meta-analysis. Journal of Cancer Survivorship 11 (6): 704-719.

Schwedhelm C, Boeing H, Hoffmann G et al. (2016) Effect of diet on mortality and cancer recurrence among cancer survivors: a systematic review and meta-analysis of cohort studies. Nutrition Reviews 74 (12): $737-$ 748. 
Scottish Government (2016) Revised Dietary Goals for Scotland. Edinburgh. Available at http://www.scotland.gov.uk/Topics/Health/Healthy-Living/Food-Health/DietaryGoalsScot (Accessed: 19 October 2019).

Stacy FG, James EL, Chapman K et al. (2015) A systematic review and meta-analysis of social cognitive theory-based physical activity and/or nutrition behaviour change interventions for cancer survivors. Journal of Cancer Survivorship 9 (2): 305-38.

Tollosa DN, Tavener M, Hure A et al. (2019) Adherence to multiple health behaviours in cancer survivors: a systematic review and meta-analysis. Journal of Cancer Survivorship 13 (3): 327-343.

Vosnaki K (2019) Chapter 3: Alcohol. In: McLean J \& Dean L (editors) The Scottish Health Survey 2018 edition. Volume 1. Main Report. The Scottish Government. Available at https://www.gov.scot/publications/scottish-health-survey-2018-volume-1-main-report/ (accessed 13 October 2019).

Wang Z, McLoone P \& Morrison DS (2015) Diet, exercise, obesity, smoking and alcohol consumption in cancer survivors and the general population: a comparative study of 16282 individuals. British Journal of Cancer 112 (3): 572-575.

WCRF/AICR (2018a) Diet, Nutrition, Physical Activity and Cancer: a Global perspective. Continuous Update Project Expert Report. Available at http://www.dietandcancerreport.org (accessed 5 October 2019).

WCRF/AICR (2018b) Continuous Update Project Expert Report 2018. Diet, nutrition, physical activity and breast cancer survivors. Available at http://www.dietandcancerreport.org (accessed 6 October 2019).

WCRF INTERNATIONAL (2018) Diet, nutrition, body composition, physical activity and cancer: regular grant programme 2018/2019. Guidelines for research grant applications and award recipients. Available at https://www.wcrf.org/sites/default/files/grant-guidelines-2018.pdf (accessed 24 August 2018). 
Table 1 Recommendations for cancer prevention (WCRF/AICR 2018a)

\section{Recommendation}

$1 \quad$ Be a healthy weight

2 Be physically active

3 Eat a diet rich in wholegrains, vegetables, fruit and beans

4 Limit consumption of 'fast foods' and other processed foods high in fat, starches or sugars

$5 \quad$ Limit consumption of red and processed meat

$6 \quad$ Limit consumption of sugar sweetened drinks

7 Limit alcohol consumption

8 Do not use supplements for cancer prevention

9 For mothers: breastfeed your baby, if you can

10 After a cancer diagnosis: follow our recommendations, if you can 
Table 2 Outline of the two-day course ‘EatWell@CLAN'

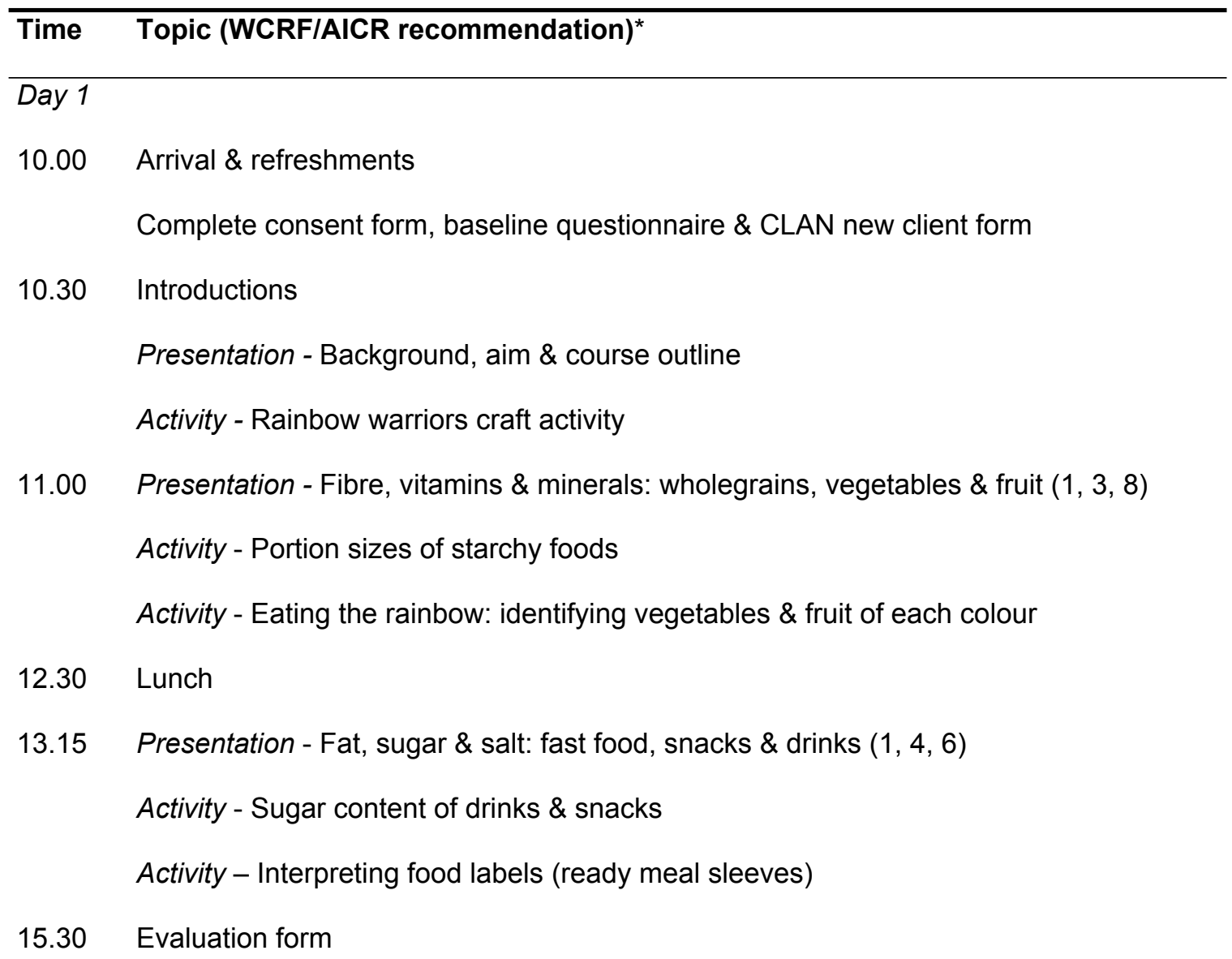

Day 2

10.00 Arrival \& refreshments

10.30 Day 1 review and discussion of key messages and any changes adopted

11.00 Presentation - Protein: meat, fish, eggs, dairy and alternatives (5)

Activity - Red meat or processed meat?

Activity - Oily fish or white fish?

12.30 Lunch

13.15 Presentation - Alcohol (7)

Activity - Units and measures of various alcoholic drinks

14.30 Presentation - Physical activity \& sedentary behaviour (2)

Activity - Talking wall: barriers \& solutions to being more active

15.20 Evaluation form

*Number relates to recommendation in table 1 
Table 3 Participant characteristics

\begin{tabular}{|c|c|c|}
\hline Characteristic & & Number (\%) \\
\hline \multirow[t]{2}{*}{ Sex } & Male & $2(18 \%)$ \\
\hline & Female & $9(82 \%)$ \\
\hline \multirow[t]{4}{*}{ Age range (years) } & $45-54$ & $3(27 \%)$ \\
\hline & $55-64$ & $4(36 \%)$ \\
\hline & $65-74$ & $3(27 \%)$ \\
\hline & $75+$ & $1(9 \%)$ \\
\hline \multirow[t]{7}{*}{ Year of first cancer diagnosis } & $2000-02$ & $1(9 \%)$ \\
\hline & 2003-05 & 0 \\
\hline & $2006-08$ & $2(18 \%)$ \\
\hline & $2009-11$ & $1(9 \%)$ \\
\hline & $2012-14$ & 0 \\
\hline & $2015-17$ & $4(36 \%)$ \\
\hline & 2018-19 & $3(27 \%)$ \\
\hline \multirow[t]{3}{*}{ Site of first cancer diagnosis } & Breast & $6(55 \%)$ \\
\hline & Lymphoma & $2(18 \%)$ \\
\hline & Other & $3(27 \%)$ \\
\hline
\end{tabular}


Figure 1 Eligibility and participation

\section{Expressed interest in participating}

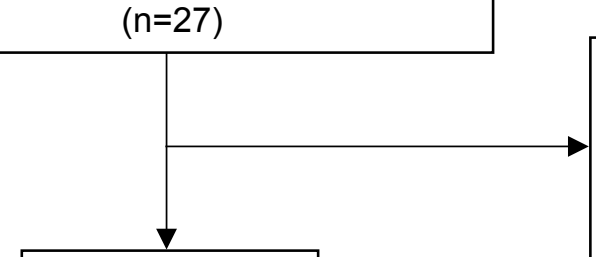

\begin{tabular}{|cc|}
\hline \multicolumn{1}{|c|}{$\begin{array}{c}\text { Not eligible } \\
(n=3)\end{array}$} \\
\\
$\begin{array}{ll}\text { Undergoing chemotherapy } & n=2 \\
\text { Not received cancer diagnosis } & n=1\end{array}$ \\
\hline
\end{tabular}

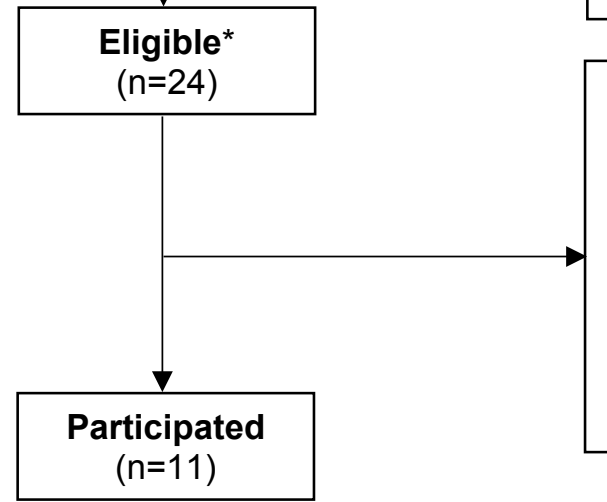

Dates of course did not suit

$n=3$

Illness

Did not want to attend alone

$n=1$

Expressed interest after course ended

$n=1$

Would feel 'out of place'

$n=1$

No reason given $\quad n=6$

${ }^{*}$ Eligibility assumed unless information given to indicate otherwise. 